\title{
Deep Learning-based Activity Detection for Grant-free Random Access
}

This paper was downloaded from TechRxiv (https://www.techrxiv.org).

LICENSE

CC BY 4.0

SUBMISSION DATE / POSTED DATE

$19-10-2021 / 21-10-2021$

CITATION

Abrao, Taufik; Souza, João Henrique Inácio de (2021): Deep Learning-based Activity Detection for Grant-free Random Access. TechRxiv. Preprint. https://doi.org/10.36227/techrxiv.16834552.v1

$\mathrm{DOI}$

10.36227/techrxiv.16834552.v1 


\title{
Deep Learning-based Activity Detection for Grant-free Random Access
}

\author{
João Henrique Inacio de Souza, Taufik Abrão
}

\begin{abstract}
The cellular internet-of-things wireless network is a promising solution to provide massive connectivity for machinetype devices. However, designing grant-free random access (GFRA) protocols to manage such connections is challenging, since they must operate in interference-aware scenarios with sporadic device activation patterns and a shortage of mutually orthogonal resources. Supervised machine learning models have provided efficient solutions for activity detection, non-coherent data detection, and non-orthogonal preamble design in scenarios with massive connectivity. Considering these promising results, in this paper, we develop two deep learning (DL) sparse support recovery algorithms to detect active devices in mMTC random access. The DL algorithms, developed to deploy GF-RA protocols, are based on the deep multilayer perceptron and the convolutional neural network models. Unlike previous works, we investigate the impact of the type of sequences for preamble design on the activity detection accuracy. Our results reveal that preambles based on the Zadoff-Chu sequences, which present good correlation properties, achieve better activity detection accuracy with the proposed algorithms than random sequences. Besides, we demonstrate that our DL algorithms achieve activity detection accuracy comparable to state-of-the-art techniques with extremely low computational complexity.
\end{abstract}

Index Terms-Massive machine-type communications (mMTC), random access protocols, grant-free access, multilayer perceptron, convolutional neural networks.

\section{INTRODUCTION}

The massive machine-type communication (mMTC) service will provide full connectivity for internet-of-thing (IoT) applications in cellular wireless networks. The machine-type devices have sporadic activation patterns, transmit small payloads with low-complexity network hardware and are powered by short lifetime batteries. At the same time, they appear as increasing massive populations distributed over wide areas. When combined, all these characteristics impose challenges to provide connectivity, especially with regard to network access. The limited channel coherence time and the high number of devices prohibit the design of mutually-orthogonal preamble sequences. Operating with fewer preambles than devices result in resource contention and network collision, which increases the access delay due to retransmissions. On the other hand, non-orthogonal preambles reduce the number of collisions, at the cost of introducing interference among the active devices and degrading the system performance [1].

This work was supported in part by the Coordenação de Aperfeiçoamento de Pessoal de Nível Superior - Brazil (CAPES) - Finance Code 001 and in part by the National Council for Scientific and Technological Development (CNPq) of Brazil under Grant 310681/2019-7.

J. H. I. de Souza and T. Abrão are with the Electrical Engineering Department, State University of Londrina, PR, Brazil. E-mail: joaohis@ outlook.com; taufik@uel.br.
The grant-free random access (GF-RA) is an alternative to provide connectivity, dealing efficiently with the interference generated by the non-orthogonal preambles, while keeping a low network access delay [2].

Methods for sparse signal processing are broadly employed to cope with the interference and enable GF-RA procedures. These techniques are suitable for the typical mMTC scenario due to the sparse nature of the signals generated by machinetype devices. Specifically, we are interested in the sparse support and signal recovery methods. Sparse support recovery aims to estimate the indices of the active devices during a random access slot transmission. In contrast, sparse signal recovery is employed for channel estimation or data detection. Approaches to perform sparse support and signal recovery are classified in compressed sensing (CS)-based, covariance (CV)based [2] and, more recently, machine learning (ML)-based.

The sparse support and signal recovery based on ML approaches have presented results which outperform substantially the other two CS-based and CV-based techniques, both in performance and computational complexity. These methods have extremely-low run time at the cost of an intense offline training phase to learn the neural network weights. Furthermore, the universal approximation theorem states that properly-designed neural networks are capable of approximate any Borel measurable function with an arbitrary non-zero amount of error [3].

We can classify the ML-based approaches into the datadriven and model-driven categories. Data-driven approaches [4]-[7] use model-agnostic schemes that learn the mapping to the outputs purely from data. These approaches are convenient for situations with intractable, incomplete, or poorly understood mathematical models, when is hard to obtain accurate parameter estimate, or when the solution results in computationally inefficient algorithms. Besides, the datadriven approaches are able to extract implicit features from data, helping to understand the problem at hand [8]. On the other hand, model-driven approaches [9]-[15] are based on the domain knowledge and structures in data. One common strategy is to craft application-specific neural networks from traditional algorithms using the deep unfolding technique originally proposed in [16]. The model-driven approaches join the specialized knowledge of classical signal processing with the flexibility and generalizability of the ML tools. However, their application need to be carefully assessed. Highly modeldependent algorithms may result in poor inference or waste of computational resources, since they ignore the fact that system parameters are dynamic and may be unknown. Additionally, algorithms which incorporate simplistic models may not cap- 
ture the main properties and dynamics of the data.

\section{A. Literature Review}

We provide an outline of the literature on sparse support and signal recovery applicable to GF-RA protocols. The approximate message passing (AMP) algorithm from the CS literature is present in many recent contributions for device activity detection and channel estimation [17], [18]. Specifically, in [18] is proposed a non-coherent scheme that embed information bits into the devices' preamble sequences, allowing joint activity and data detection. Despite the promising results of the AMP algorithm, its dependence on statistical channel information and poor stability represent technical difficulties for practical implementations.

Iterative methods using the empirical covariance matrix of the received signal are used for device activity detection in [19]. Two low-complexity algorithms to detect the set of active devices in massive MIMO unsourced random access are studied. Although the techniques achieve performance comparable to the multiple-measurement vector AMP with better numerical stability, the computational complexity is still high owing to the iterative structure of the estimation algorithms.

On the matter of the ML-based approaches, neural networks obtained via deep unfolding of existing algorithms are presented in [9]-[12], [15]. The networks of [9], [10] are based on the AMP and, differently from the original algorithm, do not require prior information about the system parameters and channel statistics due to the transformation of the AMP parameters into trainable weights. The recursive neural network obtained from the iterative shrinkage thresholding algorithm for complex group row-sparse matrix signals in [11] converges quicker while achieves more prediction accuracy than the benchmark algorithms, being robust to ill-conditioned measurement matrices. In [12] is provided a framework to develop neural networks with adaptive depth, improving the efficiency of fixed-depth networks obtained via deep unfolding.

End-to-end neural networks that mimics the noisy measurements and the sparse recovery to jointly design the preamble matrix and the receiver are proposed in [4], [6], [13]. Different deep auto-encoder algorithms, derived from CS-based and CVbased algorithms, that achieve high channel estimation and activity detection accuracy are proposed in [13]. These endto-end network designs demonstrate the benefits of correctly choosing the preamble sequences to attain reasonable sparse recovery accuracy. As demonstrated by the aforementioned works, designing preamble matrices matched with the receiver is a promising path to increase the sparse recovery accuracy. However, we remark that crafting preamble sequences from scratch results in additional overhead to transmit the generated sequences to the machine-type devices. Such problem is mitigated with the adoption of easy-to-generate sequences, which can be systematically obtained at the device-side. Considering this context, in [15] three types of easy-to-generate preamble sequences, one obtained from a Gaussian random distribution, other obtained from a binary random distribution, and the last one obtained from the deterministic Zadoff-Chu
(ZC) sequences, are evaluated and compared in joint device activity detection and channel estimation schemes. Numerical results reveal that accurate activity detection can be achieved with non-Gaussian preamble sequences taken from known sequences sets with good correlation properties.

A new algorithm for device activity detection based on a convolutional neural network (CNN) is presented in [7]. The CNN performs feature extraction from the received symbols to feed a densely connected layer that outputs the activity indicators. Simulation results reveal that the CNN outperforms a conventional neural network both in activity detection accuracy and computational complexity. Algorithms based on CNNs have been achieved promising results in applications such as image and video processing, natural language processing, and anomaly detection. Compared with the conventional densely connected layers, the convolutional layers of a CNN have sparse connections between the neurons and parameter sharing. Additionally, their computations exploit the correlation between the network inputs. In [20] is developed a framework to recover sparse signals from noisy measurements in imaging applications using a CNN. The scheme learns both the representation for signals and the inverse mapping function from measurement vectors in an end-to-end network. The proposed network approximates the solution of state-of-art algorithms in a much lower run time, achieving a remarkable tradeoff between reconstruction time and probability of successful signal recovery.

\section{B. Contributions}

In this paper, we develop two deep learning (DL) algorithms for sparse support recovery used for efficient device activity detection in mMTC GF-RA protocols. The proposed DL datadriven neural networks do not require any statistical information of the channel or the devices' activity, having extremelylow execution time and being robust to variations in parameters of the system. The first network is a deep multilayer perceptron (DMLP) composed by densely connected layers, which inputs are the received symbols during the random access slot. The second network is a CNN composed by stacked convolutional layers, which inputs are the received symbols correlated with the preamble sequences. Especially, the CNN performs the sparse support recovery by exploiting the structured correlation of the network inputs.

We extend the neural network design workflow by adding a feature selection step, where the type of the preamble sequences is selected aiming to provide input features for the networks which increase the activity detection accuracy. During this step, we evaluate the DMLP and CNN with features generated by random complex Normal and Bernoulli sequences, as well as deterministic ZC sequences [21], which present good correlation properties. We demonstrate that the feature selection, in our case, represented by the choice of preamble sequences type, is as important as the network architecture design, supporting the use of well-known and easy-to-generate sequences to integrate ML-based algorithms for applications in wireless communications.

The proposed networks are subjected to a parameters selection step aiming to optimize their architectures. Then, an 
offline training with the backpropagation algorithm and a representative data set is performed to learn the networks weights. Differently from other works in the literature, we perform a comprehensive analysis of the activity detection error via the receiver operating characteristic (ROC) and the detection error tradeoff (DET) metrics. Numerical results reveal that the DL algorithms have competitive activity detection accuracy with extremely lower execution time than stateof-art CS-based algorithms. Especially, the feature selection demonstrates that the $\mathrm{CNN}$ benefits from the good correlation properties of the $\mathrm{ZC}$ sequences, achieving improved activity detection accuracy in the mMTC scenario. Besides, the networks are robust to variations on the system parameters, demonstrating the generalization power of the DL models.

\section{SySTEM MODEL}

In this section, we describe the system model, as well as formulate the device activity detection problem. We consider the uplink of a narrow-band system constituted by one cell with $K$ machine-type devices, served by a single-antenna basestation (BS), as is depicted in Fig. 1. These devices access the network with probability $p_{a} \ll 1$. For this reason, the number of active devices is much less than the total in the cell.

During the random access slot, the active devices transmit unique preamble sequences. These sequences identify the devices and are used at the BS to detect the set of active devices. The preamble sequences have a length of $L<K$ symbols due to the limited channel coherence time. For this reason, it is impossible to design mutually-orthogonal sets of preambles. In our work, we consider three types of nonorthogonal sequences for the preambles. The non-orthogonal sequences result in interference on the transmitted signal by the set of active devices during a random access slot. Details on the used sequences are given in the next subsection. The preamble sequences are defined by the vectors $\mathbf{a}_{k} \in \mathbb{C}^{L \times 1}$ for $k=1, \ldots, K$, and are normalized such that $\left\|\mathbf{a}_{k}\right\|_{2}^{2}=L, \forall k$. The preamble matrix $\mathbf{A} \in \mathbb{C}^{L \times K}$, which is known at the $\mathrm{BS}$, contains in its columns the preamble sequences of all the $K$ devices in the cell.

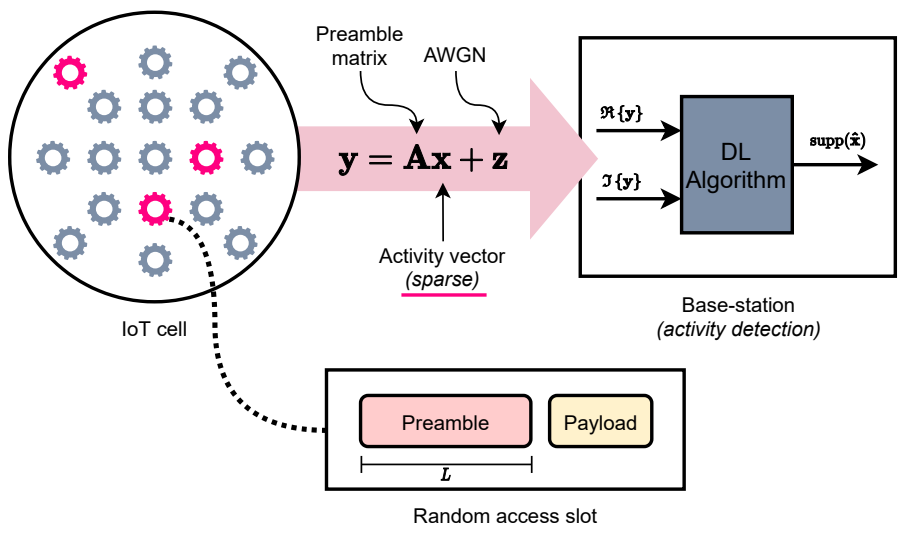

Figure 1. System diagram with the IoT communication cell, the random access slot structure, and a device activity detection scheme based on a DL algorithm.
Let $\alpha_{k} \in\{0,1\}$ for $k=1, \ldots, K$ be an activity state indicator of the device $k$, which is equal to 1 if the device is active and 0 otherwise. We consider that the probability of each device being active follows a Bernoulli distribution, i.e. $\alpha_{k} \sim p_{\alpha_{k}}(u)=\left(1-p_{a}\right) \delta(u)+p_{a} \delta(u-1), \forall k$, where $p_{\alpha_{k}}(u)$ is the probability mass function (PMF) of $\alpha_{k}$ and $\delta(u)$ is the Dirac delta function. The vector with the activity state indicators of all the $K$ devices is named activity descriptor,

$$
\boldsymbol{\alpha}=\left[\begin{array}{lll}
\alpha_{1} & \cdots & \alpha_{K}
\end{array}\right]^{T}
$$

The channel links between each device and the BS follow the Rayleigh fading model, with channel coefficients associated to the device $k$ defined by $h_{k} \sim \mathcal{C N}(0,1)$. It is worth noting that the evaluation of the proposed deep learningbased solutions under different channel conditions, e.g., lineof-sight propagation or spatially-correlated channel vectors, is a promising path for future investigation.

The received signal at the BS during the random access slot is equal to the superposition of the preambles transmitted by each active device, which is written as

$$
\mathbf{y}=\sum_{k=1}^{K} \alpha_{k} h_{k} \mathbf{a}_{k}+\mathbf{z}
$$

where $\mathbf{z} \sim \mathcal{C N}\left(\mathbf{0}, \sigma_{z}^{2} \mathbf{I}\right)$ is the additive white Gaussian noise. Letting $\mathbf{x}=\left[\begin{array}{lll}\alpha_{1} h_{1} & \cdots & \alpha_{K} h_{K}\end{array}\right]^{T}$ allows us to write the compact form of the received signal

$$
\mathbf{y}=\mathbf{A x}+\mathbf{z}
$$

The goal of the device activity detection problem is to determine the set of active devices from the received signal $\mathbf{y}$ and the preamble matrix $\mathbf{A}$. This is equivalent to finding the index of the non-zero entries of the vector $\mathbf{x}$, namely the support of $\mathbf{x}$,

$$
\operatorname{supp}(\mathbf{x})=\left\{k \in \mathbb{N}_{+} \mid x_{k} \neq 0\right\}
$$

As the number of active devices is much less than the total number of devices in the cell (sporadic activation, i.e. $p_{a} \ll 1$ ), $\mathbf{x}$ has a sparse structure. For this reason, the problem of finding the support of $\mathbf{x}$ from $\mathbf{y}$ and $\mathbf{A}$ can be cast as a sparse support recovery problem.

The sparse support recovery problem is an object of study of the compressed sensing field, and have been addressed in many works using different approaches, e.g. see [22] and the references therein. In our work, the aim is to develop two DL algorithms for sparse support recovery in the context of device activity detection for mMTC. We demonstrate that a DL algorithm which inputs are the real and imaginary parts of the received signal is sufficient to perform device activity detection efficiently.

\section{A. Non-orthogonal Preamble Sequences}

The preamble sequences play a key role in the device activity detection problem, as they dictate the interference patterns on the signal transmitted by the active devices during each random access slot. In addition, the inputs of the activity detection DL algorithms developed in the sequence are based 
on the BS received symbols in eq. (3), a linear transformation of the preamble matrix. Hence, the learned neural network weights depend directly on the preamble sequences, which controls how the signals of the simultaneously active devices are interrelated.

We remark two desired properties in preamble sequences. The preamble sequences must be easy-to-generate, coordinately, at the device-side. In the massive machine-type GF-RA, a prohibitive communication overhead is required to transmit fully-learned preamble matrices, as the ones proposed in [4], [6], [13], to the high number of devices in the cell. Conversely, well-known sequences as the $\mathrm{ZC}$ and random sequences can be generated systematically and coordinately at the device-side with low overhead. The second property is the separability among the sequences in the set. The separability governs the interference levels between the signals of the simultaneously active devices. For this reason, choosing sets of sequences with high separability is important to obtain reasonable activity detection accuracy.

In the context of sparse support recovery using CS techniques, it is common to use preambles generated by sampling random distributions. However, we show that DL algorithms with deterministic preamble sequences that have good correlation properties can achieve reasonable activity detection accuracy. In the following, we present three suitable types of preamble sequences used in our work.

Random Sequences. We use two types of random sequences for the preambles. Random sequences are useful, specially in cases where the number of mutually orthogonal preambles is not sufficient for all devices, e.g. in crowded mMTC applications, because it is easy to generate large amount of unique preambles. Additionally, matrices generated by sampling sub-Gaussian random distributions (e.g. Normal and Bernoulli) satisfy, with high probability, the restricted isometry property (RIP), a necessary condition to guarantee the signal reconstruction of many CS algorithms [23]. The first type of random sequence is obtained by sampling a circularlysymmetric complex Normal distribution with zero mean and unitary variance:

$$
\left[\overline{\mathbf{a}}_{k}^{\mathrm{G}}\right]_{l} \sim \mathcal{C N}(0,1)
$$

for $k=1, \ldots, K$ and $l=1, \ldots, L$. We name this type of sequence as Normal. In order to met the preamble normalization presented previously, we scale the Normal preamble with the factor:

$$
\mathbf{a}_{k}^{\mathrm{G}}=\sqrt{\frac{L}{\left\|\overline{\mathbf{a}}_{k}^{\mathrm{G}}\right\|_{2}^{2}}} \overline{\mathbf{a}}_{k}^{\mathrm{G}}
$$

The second type of random sequence type is the Bernoulli. The Bernoulli sequences are real two-valued sequences obtained by sampling a symmetric random distribution of the type

$$
\left[\mathbf{a}_{k}^{\mathrm{B}}\right]_{l} \sim p_{\mathrm{B}}(u)=\frac{1}{2} \delta(u+1)+\frac{1}{2} \delta(u-1)
$$

for $k=1, \ldots, K$ and $l=1, \ldots, L$. As each entry of the Bernoulli sequence has norm equal to 1 , normalization is not required.
Zadoff-Chu Sequences. The ZC sequence is a type of polyphase sequence which entries are defined by [21]:

$$
\left[\overline{\mathbf{a}}_{r}^{\mathrm{zC}}\right]_{l}=\exp \left[-\frac{j \pi r}{L}(l-1) l\right], l=1, \ldots, L
$$

where $j=\sqrt{-1}$ and $r \in\{1, \ldots, L-1\}$ is a number relatively prime to $L$ named the sequence root. A $\mathrm{ZC}$ sequence has the ideal auto-correlation property, i.e. its auto-correlation value is equal to zero for all shifts other than zero. For this reason, a sequence and its shifted versions comprise a set of mutually-orthogonal sequences. The ideal auto-correlation property holds only for sequences generated by a single root. On the other hand, sequences generated by different roots have constant cross-correlation equal to $\sqrt{L}$ if the difference between the roots is relatively prime to $L$ [24]. Therefore, a set of non-orthogonal sequences with a three-valued crosscorrelation function can be generated by taking the shifted versions of multi-root $\mathrm{ZC}$ sequences. We use this set of nonorthogonal sequences to generate the $\mathrm{ZC}$ preambles. The $\mathrm{ZC}$ preambles are defined by the sequences

$$
\overline{\mathbf{a}}_{r}^{\mathrm{ZC}}, \forall r \in \mathcal{R}
$$

and its shifted versions, considering $\mathcal{R} \subset\{1, \ldots, L-1\}$ the set of chosen roots. Given the number of devices in the cell and the preamble length, the minimum number of roots to generate unique preambles for all the $K$ devices is equal to

$$
N_{r}=\left\lceil\frac{K}{L}\right\rceil
$$

It is worth mentioning that the set of non-orthogonal $\mathrm{ZC}$ preambles is composed by $|\mathcal{R}| \geq N_{r}$ smaller sub-sets of orthogonal ones. For this reason, allocating the sub-set of orthogonal preambles to devices with similar activation pattern is an efficient alternative to manage the interference levels.

\section{B. Sequences Performance in Activity Detection Problem}

In order to give an insight of the impact of the preamble design on the performance of the device activity detection scheme, we demonstrate a result on the distribution of the signal-to-interference-plus-noise (SINR) ratio of the received signal at the BS for the analyzed sequence types. The SINR gives an indirect measure of the activity detection accuracy, as it demonstrates the ratio between the power of the signal of an active device and the power of noise plus the interference generated by the simultaneously interfering devices. Fig. 2 depicts the empirical cumulative distribution function (CDF) of the SINR for the $k$-th device by using the Normal, Bernoulli, and $\mathrm{ZC}$ sequences, considering three values of activation probability, $p_{a} \in\{0.01,0.10,0.30\}$. The remain setup parameter values to generate this result are $K=40$, $L=20$, and $\sigma_{z}^{2}=0.1$, resulting in a signal-to-noise ratio (SNR) of $10 \mathrm{~dB}$. Besides, we define the SINR correlating the received signal at the $\mathrm{BS}$ with the preamble of each active device, resulting:

$$
\operatorname{SINR}_{k}=\frac{\left\|\mathbf{a}_{k}\right\|_{2}^{4}\left|h_{k}\right|^{2}}{\sum_{i \in \mathcal{K}_{\mathrm{A}}, i \neq k}\left|\mathbf{a}_{k}^{H} \mathbf{a}_{i}\right|^{2}\left|h_{i}\right|^{2}+\left|\mathbf{a}_{k}^{H} \mathbf{z}\right|^{2}}
$$




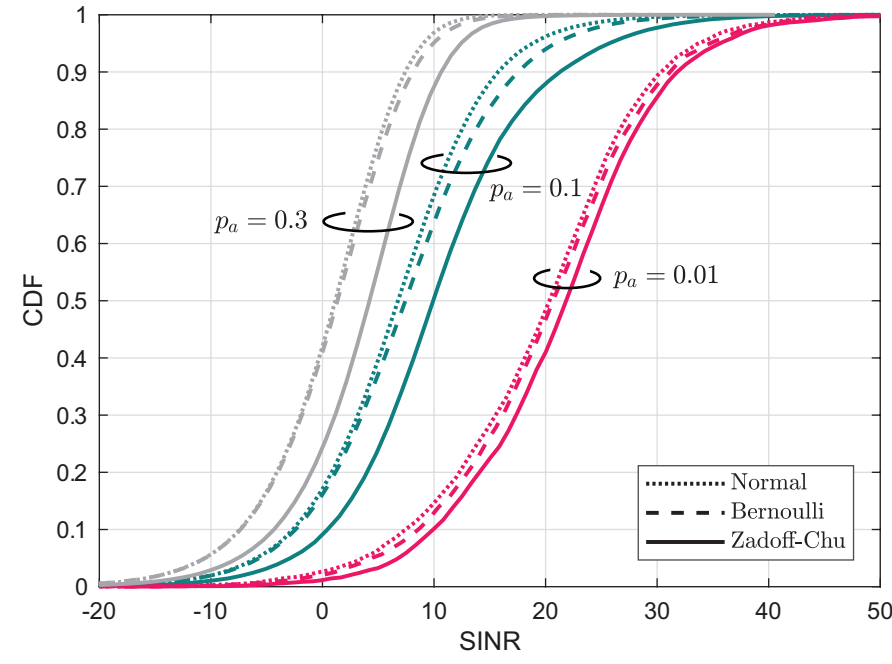

Figure 2. Empirical CDF of the SINR achieved by Normal, Bernoulli, and $\mathrm{ZC}$ sequences for three values of $p_{a}$.

for $k \in \mathcal{K}_{\mathrm{A}}$, where $\mathcal{K}_{\mathrm{A}}$ is the set of active devices during a specific random access slot. From Fig. 2, one can observe in the set of curves that decreasing the activation probability increases the average SINR values, since it reduces the number of simultaneous interfering devices during a specific transmission interval. The $\mathrm{ZC}$ sequences achieve the best average SINR values due to their good correlation properties. At the same time, the Bernoulli sequences outperform marginally the Normal ones in terms of the SINR distribution.

\section{DEEP LEARNING AlgorithMS}

In this section, we introduce the architectures of the DMLP and CNN algorithms devised for device activity detection. The two DL algorithms exploit different input features to perform device activity detection. Especially, the structure of the CNN exploits the correlation between the network inputs, benefiting from the good correlation properties of the ZC sequences for preamble design. The DL algorithms are represented by functions of the type $f\left(\mathbf{v}_{0}, \boldsymbol{\theta}\right): \mathcal{I} \rightarrow \mathbb{R}^{K \times 1}$, which learns an inverse map of the activity descriptor from the signal received during the random access slot. In these functions, we consider $\mathbf{v}_{0} \in \mathcal{I}$ the inputs in the input space $\mathcal{I}$, and $\boldsymbol{\theta}$ a set with the algorithm parameters. The learning phase is implemented by a training procedure, in which the parameters $\boldsymbol{\theta}$ of the function $f\left(\mathbf{v}_{0}, \boldsymbol{\theta}\right)$ are adjusted following a set of examples containing the activity descriptor and the respective received signal at the BS.

Let $\left\{\left(\mathbf{y}^{(s)}, \boldsymbol{\alpha}^{(s)}\right) \mid s=1, \ldots, S\right\}$ be a set of samples containing the received signal and the activity descriptor of a random access slot. We denote the output of a DL algorithm for the sample $s$ as $\tilde{\boldsymbol{\alpha}}^{(s)}$. Taking this into account, we can measure the quality of the estimate $\tilde{\boldsymbol{\alpha}}^{(s)}$ w.r.t. the original activity descriptor $\boldsymbol{\alpha}^{(s)}$ by calculating the binary cross-entropy function. In our DL algorithms, the loss function is defined as the average binary cross-entropy function [25], calculated over all the $S$ samples as:

$$
\begin{aligned}
\mathcal{L}=\frac{1}{S K} \sum_{s=1}^{S} \sum_{k=1}^{K}- & {\left[\alpha_{k}^{(s)} \log \left(\tilde{\alpha}_{k}^{(s)}\right)+\right.} \\
& \left.\left(1-\alpha_{k}^{(s)}\right) \log \left(1-\tilde{\alpha}_{k}^{(s)}\right)\right]
\end{aligned}
$$

The aim of the training procedure is to minimize $\mathcal{L}$ by choosing the right parameters of $f\left(\mathbf{v}_{0}, \boldsymbol{\theta}\right)$. In this way, the trained DL network produces an accurate estimate of the activity descriptor.

\section{A. Deep Multilayer Perceptron}

The DMLP algorithm, as depicted in Fig. 3, is implemented with densely connected layers. Its inputs are the real and imaginary parts of the received signal, while the output is an estimate of the activity descriptor. In the following, we describe in detail the layers of the DMLP.

The DMLP architecture has two densely connected hidden layers with $N_{n}$ neurons and one densely connected output layer with $K$ neurons. The hidden layers have the ReLU activation function:

$$
\operatorname{ReLU}(u)=\max (0, u)
$$

On the other hand, the output layer has the sigmoid activation function:

$$
\operatorname{Sigmoid}(u)=\frac{1}{1+\exp (-u)}
$$

The details of the DMLP layers are organized in Table I. It is worth noting that the number of inputs and outputs of DMLP are tied respectively to the preamble length, $L$, and the number of devices $K$. Hence, one needs to re-train a network from scratch in order to accommodate different numbers of devices with different preamble lengths.

Now, we introduce the parameters of the DMLP layers, which are the weights matrices and the bias values vectors. Given the dimensions of the inputs and outputs of each layer, we have the weights matrices $\mathbf{W}_{1} \in \mathbb{R}^{N_{n} \times 2 L}, \mathbf{W}_{2} \in$ $\mathbb{R}^{N_{n} \times N_{n}}$ and $\mathbf{W}_{3} \in \mathbb{R}^{K \times N_{n}}$ for the hidden layers 1 and 2 , and

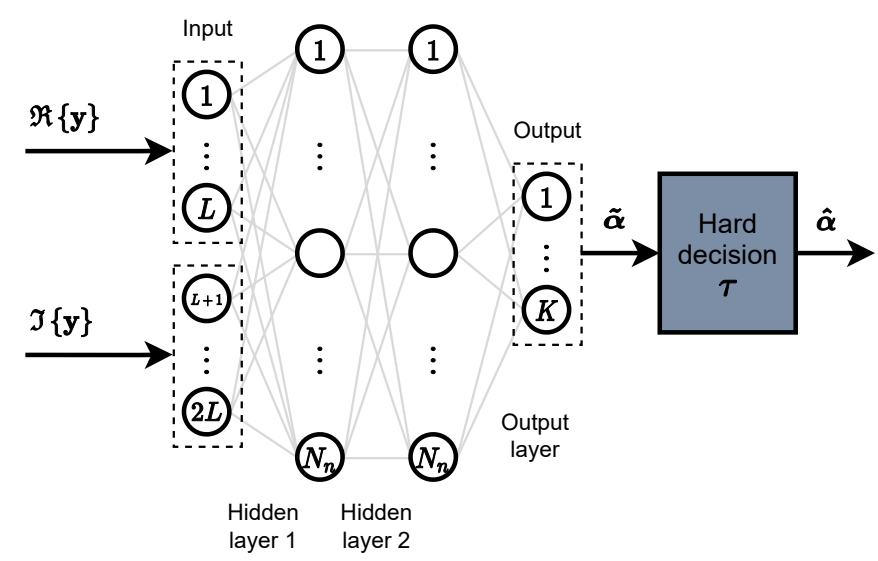

Figure 3. Architecture of the DMLP algorithm. proposed for device activity detection. The inputs are the real and imaginary parts of the received signal, while the outputs is an estimate of the activity descriptor. 
Table I

DESCRIPTION OF THE DMLP ALGORITHM LAYERS.

\begin{tabular}{|l|c|c|c|}
\hline Layer & Type & Activation function & Neurons \\
\hline Hidden 1 & Densely connected & ReLU & $N_{n}$ \\
Hidden 2 & Densely connected & ReLU & $N_{n}$ \\
Output & Densely connected & Sigmoid & $K$ \\
\hline
\end{tabular}

the output layer, respectively. Similarly, the vectors with the bias values are $\mathbf{b}_{1} \in \mathbb{R}^{N_{n} \times 1}, \mathbf{b}_{2} \in \mathbb{R}^{N_{n} \times 1}$ and $\mathbf{b}_{3} \in \mathbb{R}^{K \times 1}$. Taking this into account, we define the set with the DMLP parameters by

$$
\boldsymbol{\theta}_{\mathrm{DMLP}}=\left\{\mathbf{W}_{i}, \mathbf{b}_{i}\right\}_{i=1}^{3}
$$

Considering the DMLP parameters, we write the inputoutput relation of the hidden layers in the DMLP:

$$
\mathbf{v}_{i}=\operatorname{ReLU}\left(\mathbf{W}_{i} \mathbf{v}_{i-1}+\mathbf{b}_{i}\right), i=1,2
$$

where $\mathbf{v}_{i}$ is the layer output, considering $\mathbf{v}_{0}$ as the DMLP input, and the activation function is calculated for each entry of the input vector. The input-output relation of the output layer is obtained following the same logic, substituting the layer parameters, as well as the ReLU activation by the sigmoid function.

With $\boldsymbol{\theta}_{\mathrm{DMLP}}$ in hands, it is possible to calculate the number of trainable parameters in the DMLP algorithm. Considering the dimensions of the weights matrices and the bias vectors values, the number of trainable parameters is given by:

$$
\Theta_{\text {DMLP }}\left(N_{n}, L, K\right)=N_{n}^{2}+2 N_{n} L+N_{n} K+2 N_{n}+K
$$

Since the outputs of the DMLP are in the range $[0,1]$, a hard decision module with threshold parameter $\tau \geq 0$ is positioned at the output of the algorithm to calculate the activity descriptor in its original domain. Hence, the hard decision output is:

$$
\hat{\alpha}_{k}= \begin{cases}0, & \tilde{\alpha}_{k}<\tau \\ 1, & \tilde{\alpha}_{k} \geq \tau\end{cases}
$$

During the device activity detection, two different kinds of error may occur. First, a false alarm (FA) occur when an inactive device is detected as active. Second, a miss detection (MD) occur when an active device is detected as inactive. The probabilities of FA and MD are calculated in terms of the hard decision threshold by:

$$
P_{\mathrm{FA}}(\tau)=\operatorname{Pr}\left(\tilde{\alpha}_{k} \geq \tau \mid \alpha_{k}=0\right)
$$

$$
P_{\mathrm{MD}}(\tau)=\operatorname{Pr}\left(\tilde{\alpha}_{k}<\tau \mid \alpha_{k}=1\right)
$$

Given the imbalance on the number of active devices during each random access slot, the frequency of each type of error changes. The error probability as a function of $\tau$ is written in terms of the FA and MD probabilities as

$$
P_{\mathrm{E}}(\tau)=\left(1-p_{a}\right) P_{\mathrm{FA}}(\tau)+p_{a} P_{\mathrm{MD}}(\tau)
$$

The hard decision module can be optimized in order to meet different design criteria, e.g. minimize a specific type of error, or a metric which combines the two types of error with different weights. In our work, we evaluate the algorithms adjusting the hard decision thresholdsuch that

$$
\tau^{*}=\underset{\tau \geq 0}{\arg \min } P_{\mathrm{E}}(\tau)
$$

\section{B. Convolutional Neural Network}

Now, we introduce the CNN algorithm depicted in the Fig. 4. Differently from the DMLP, the CNN algorithm is implemented with convolutional layers. Particularly, we need to adjust the input dimension aiming to match it with the number of outputs. In order to accomplish this while exploiting the good correlation properties of the $\mathrm{ZC}$ preamble sequences, we use a correlator stage at the input of the network, which produces the signal

$$
\mathbf{y}_{\text {corr }}=\mathbf{A}^{H} \mathbf{y}
$$

This correlator stage can be seen as an additional layer with fixed weights instead of trainable parameters. Then, the inputs of the $\mathrm{CNN}$ are the real and imaginary parts of the correlated received signal, organized as two vectors of dimension $K \times 1$. The output is an estimate of the activity descriptor.

The CNN architecture has two hidden layers of the type 1D convolution with $N_{f}$ feature maps each one, filters of length $N_{w}$ and ReLU as the activation functions. The output layer has one feature map, filters of length $N_{w}$ and sigmoid as the activation function. The details on the layers of the CNN algorithm are described in Table II. From Table II, we see that the CNN architecture do not depends directly on system parameters like the number of devices or the preamble length. In addition, differently from the DMLP, the CNN has a flexible structure that allows inputs of variable size due to the windowbased computations across the layers. Hence, a trained CNN can be reused for other system configurations by changing properly the correlator stage.

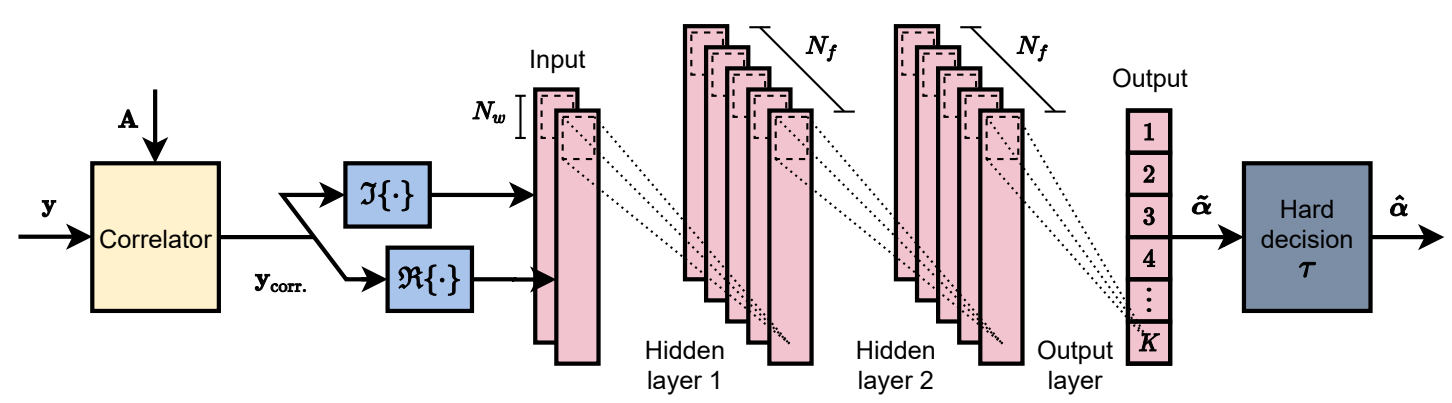

Figure 4. Architecture of the CNN algorithm, proposed for device activity detection. The inputs of the convolutional layers are the real and imaginary parts of the correlated received signal, while the network output is an estimate of the activity descriptor. 
Table II

DESCRIPTION OF THE CNN ALGORITHM LAYERS.

\begin{tabular}{|l|c|c|c|c|}
\hline Layer & Type & Activation & Feature maps & Filter length \\
\hline Hidden 1 & 1D Conv & ReLU & $N_{f}$ & $N_{w}$ \\
Hidden 2 & 1D Conv & ReLU & $N_{f}$ & $N_{w}$ \\
Output & 1D Conv & Sigmoid & 1 & $N_{w}$ \\
\hline
\end{tabular}

Conv: convolution.

The parameters of the CNN layers are the filters and the bias values w.r.t. each feature map. Let the superscript $\ell$ be the index of the feature map. The parameters of the first hidden layer are $\mathbf{W}_{1}^{\ell} \in \mathbb{R}^{N_{w} \times 2}$ and $b_{1}^{\ell} \in \mathbb{R}, \ell=1, \ldots, N_{f}$. Next, the parameters of the second hidden layer are $\mathbf{W}_{2}^{\ell} \in \mathbb{R}^{N_{w} \times N_{f}}$ and $b_{2}^{\ell} \in \mathbb{R}, \ell=1, \ldots, N_{f}$. Finally, the parameters of the output layer are $\mathbf{W}_{3}^{1} \in \mathbb{R}^{N_{w} \times N_{f}}$ and $b_{3}^{1} \in \mathbb{R}$. With these definitions, we assume the set with the CNN parameters as:

$$
\boldsymbol{\theta}_{\mathrm{CNN}}=\left\{\left\{\mathbf{W}_{1}^{\ell}, b_{1}^{\ell}\right\}_{\ell=1}^{N_{f}},\left\{\mathbf{W}_{2}^{\ell}, b_{2}^{\ell}\right\}_{\ell=1}^{N_{f}},\left\{\mathbf{W}_{3}^{1}, b_{3}^{1}\right\}\right\}
$$

Given the respective dimensions of the CNN parameters, we calculate the number of trainable parameters in the algorithm by the equation:

$$
\Theta_{\mathrm{CNN}}\left(N_{f}, N_{w}\right)=N_{f}^{2} N_{w}+3 N_{f} N_{w}+2 N_{f}+1
$$

It is important to stress that, differently from the DMLP, the number of trainable parameters in the $\mathrm{CNN}$ does not depend explicitly on the number of devices and the preamble length. This is a good advantage of the CNN, as its size do not grow rapidly with the scenario parameters. However, $N_{f}$ and $N_{w}$ must follow the changes of the parameters in the scenario in order to achieve efficient results on device activity detection.

$$
[\mathbf{M} * \mathbf{N}]_{t}=\sum_{i=t-n}^{t-1} \overline{\mathbf{M}}_{i,:} \mathbf{N}_{t-i,:}^{T}
$$

where $\overline{\mathbf{M}} \in \mathbb{R}^{n \times p}$ is a zero-padded version of $\mathbf{M}$. The inputoutput relation of the hidden layers is given by the equation:

$$
\left[\mathbf{v}_{i}^{\ell}\right]_{t}=\operatorname{ReLU}\left(\left[\mathbf{W}_{i}^{\ell} * \mathbf{V}_{i-1}\right]_{t}+b_{i}^{\ell}\right)
$$

for $i=1,2$ and $\ell=1, \ldots, N_{f}$. We consider $\mathbf{v}_{i}^{\ell}$ as the output of the $\ell$-th feature map in the layer $i$, while $\mathbf{V}_{i}$ is a matrix which columns are the output vectors of all the feature maps, or channels, in the layer $i$,

$$
\mathbf{V}_{i}=\left[\begin{array}{lll}
\mathbf{v}_{i}^{1} & \cdots & \mathbf{v}_{i}^{N_{f}}
\end{array}\right]
$$

The input-output relation of the output layer can be obtained easily by substituting the layer parameters and the ReLU activation by the sigmoid. As the DMLP, the CNN has a hard decision module to calculate the estimate of the activity descriptor in the original domain.

\section{NumericAl RESUlts}

In this section, we cover the numerical results associated to the proposed DL algorithms and the preambles design, as well as the baseline device activity detection techniques.

\section{A. DL Training Procedure}

The steps to train both DMLP and CNN algorithms for solving the device activity detection problem in mMTC scenarios are described herein. First, observe that a sample represents a transmission interval, storing the real and imaginary parts of the received signal, as well as the devices activity descriptor. We create the samples generating the activity descriptor and evaluating eq. (3). It is worth mentioning that we generate all the signals by sampling random distributions following the definitions in Section II. A data set is constituted by $5 \cdot 10^{5}$ samples, from which $4.5 \cdot 10^{5}$ are used for training and $5 \cdot 10^{4}$ used for validation. Each data set is generated with a single realization of the preamble matrix. The DL algorithms are trained with the data set samples using the adaptive moment (ADAM) estimation algorithm. The training stops if the loss function $\mathcal{L}$ in eq. (12) do not improve after 5 epochs. Table III contains the information on the training setup of the DL algorithms.

Table IV contains the average training time of the proposed DL algorithms for different preamble lengths. The training is performed on a workstation equipped with a Nvidia GeForce 940MX GPU, an Intel(R) Core(TM) i5-7200U CPU @ 2.5 $\mathrm{GHz}$ and $8 \mathrm{~GB}$ of RAM.

\section{B. DL Input Parameters Tuning}

In the following, we describe the procedure to tune the architectural parameters of the DL algorithms. In order to get high activity detection accuracy and prevent overfitting, we carefully tune the number of neurons in the hidden layers of the DMLP and the number of feature maps in the hidden layers of the CNN. The parameters are tuned by hand in the the ranges $N_{n} \in\{80,160,320,400,800,1600\}$ and $N_{f} \in\{20,40,80\}$. The tuning procedure consists on generating and assessing the learning curves of the algorithms, which contain the loss values along the epochs, calculated by the binary cross-entropy function in eq. (12) on the training and validation data sets. Ideally, the loss values calculated for the validation set must follow the values calculated for the training one, indicating improvement on the generalization capability of the algorithm. If the validation loss worsens, or

Table III

TRAINING SETUP USED FOR THE DL ALGORITHMS VIA ADAM.

\begin{tabular}{|l|l|}
\hline Training algorithm & ADAM estimation \\
\hline Training samples & $4.5 \cdot 10^{5}(90 \%)$ \\
\hline Validation samples & $0.5 \cdot 10^{5}(10 \%)$ \\
\hline Batch size & 128 \\
\hline Maximum epochs & $10^{5}$ \\
\hline Epochs for early stopping & 5 \\
\hline
\end{tabular}

Table IV

TRAINING TIME (SECONDS) OF THE DL ALGORITHMS ACCORDING TO THE PREAMBLE LENGTH.

\begin{tabular}{|c|c|c|}
\hline$L$ & DMLP & CNN \\
\hline 7 & $2.49 \cdot 10^{2}$ & $1.25 \cdot 10^{3}$ \\
13 & $3.88 \cdot 10^{2}$ & $1.72 \cdot 10^{3}$ \\
17 & $3.97 \cdot 10^{2}$ & $1.78 \cdot 10^{3}$ \\
21 & $3.82 \cdot 10^{2}$ & $1.38 \cdot 10^{3}$ \\
\hline
\end{tabular}


the gap between the losses of the two data sets increases, there is no more gain on increasing the parameter value.

Fig. 5 depicts the learning curves for the DL algorithms varying their architectural parameters ${ }^{1}$. Analyzing the curves, we observe that the minimum training loss always reduces increasing the parameters value, as expected. Fig. 5(a) illustrates the learning curves of the DMLP for $N_{n} \in\{80,160,320\}$, while the Fig. 5(c) depicts the validation loss for $N_{n} \in$ $\{320,400,800\}$. Combining these results, one can infer that the DMLP attains overfitting with $N_{n}>400$. Furthermore, we observe that the validation loss improves marginally from $N_{n}=320$ to $N_{n}=400$. Hence, we choose $N_{n}=320$ for the DMLP algorithm. On the other hand, the validation loss of the CNN, depicted in Fig. 5(b), always decreases with the epochs. Despite that, the gap between the validation and training losses increases for $N_{f}>40$. Considering these facts, we choose $N_{f}=40$ for the CNN.

\section{Performance: Error rate, FA and $M D$ rates}

Numerical results on the activity detection accuracy of the proposed DL activity detection algorithms for GF-RA protocols are assessed and compared with two baseline algorithms available in the literature, the least absolute shrinkage and selection operator (LASSO) method [26] and the AMP algorithm [27]. The adopted AMP algorithm is implemented with

\footnotetext{
${ }^{1}$ We omit a few curves in order to preserve the readability of the result.
}

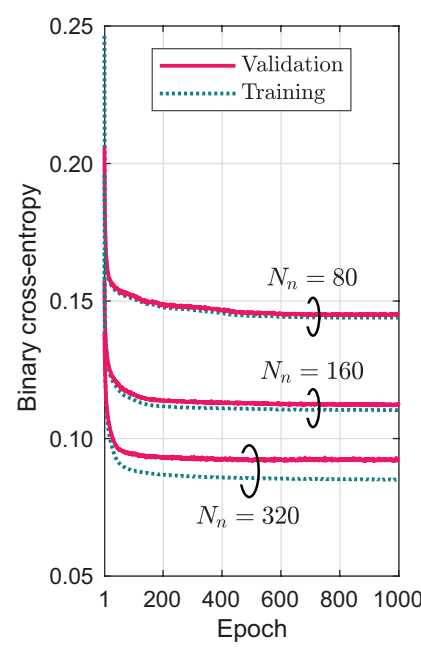

(a) DMLP varying $N_{n}$

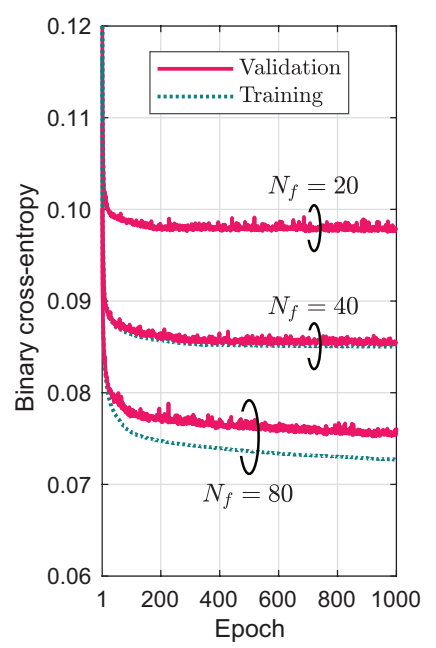

(b) $\mathrm{CNN}$ varying $N_{f}$

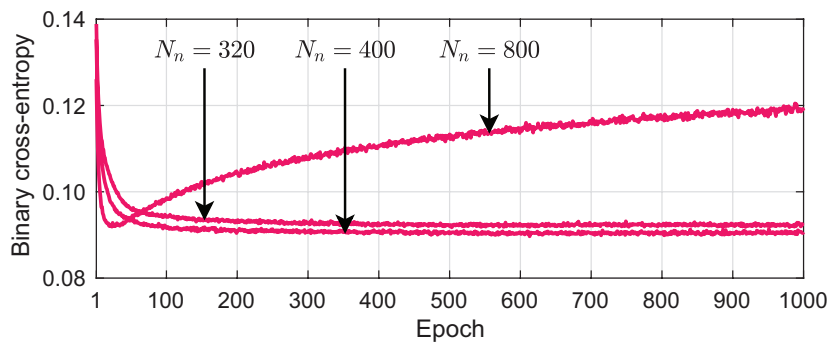

(c) Demonstration of overfitting in the DMLP

Figure 5. Learning curves with the training and validation losses (binary cross-entropy) vs. the epochs of the DL algorithms, varying their architectural parameters. the complex soft thresholding denoising function [28], while the LASSO method is computed via the coordinate descent optimization (CD) algorithm [29]. Both thresholding factor of AMP and regularization parameter of the LASSO are tuned for each evaluated scenario in order to develop a fair comparison, in which the baseline algorithms achieve good performancecomplexity tradeoffs. Three figure of merit are evaluated: error rate, false alarm rate, and miss detection rate. Table $\mathrm{V}$ summarizes the setup used to produce the evaluated scenarios and respective numerical results. The scenario parameters are consistent with other recently published papers on ML-based approaches for GF-RA [4], [6]. In addition, all the signals are generated by sampling random distributions following the definitions in Section II.

Fig. 6 depicts the error rate vs. the undersampling ratio $\Delta=L / K$ for both DL algorithms, as well as the baseline algorithms. We evaluate each DL algorithm using the three types of sequences. The first observation is that the detection error rate improves by increasing $\Delta$, as the longer preamble sequences provide more information to the activity detection algorithms. The DMLP algorithm using random sequences achieves better performance than the $\mathrm{CNN}$ under the same condition. However, the $\mathrm{CNN}$ with the $\mathrm{ZC}$ sequences achieves detection error rate values comparable to the DMLP. The CNN has a huge improvement in detection error rate with the $\mathrm{ZC}$ sequences instead of the random ones. This is due the correlator stage in the $\mathrm{CNN}$ combined with the good correlation properties of the $\mathrm{ZC}$ sequences. In addition, the structure of $\mathrm{CNN}$ exploit the correlation among the samples of the network inputs. The activity detection accuracies for the CNN with the two random sequences are comparable, while the differences on the error rate of the DMLP with each type of sequences are non-negligible. Besides, comparing with the baseline algorithms, the DMLP achieves error rate levels comparable to the AMP for $\Delta \geq 0.325$. On the other hand, considering low undersampling ratios, i.e., $\Delta \leq 0.175$

Table V

SIMULATION PARAMETERS.

\begin{tabular}{|l|l|}
\hline Parameter & Value \\
\hline \multicolumn{2}{|c|}{ System } \\
\hline Number of devices & $K=40$ \\
Activation probability & $p_{a} \in[0.01,0.3]$ \\
Type of sequences & Normal, Bernoulli, ZC [21], [24] \\
Preamble length & $L \in\{7,13,17,21\}$ \\
Undersampling ratio & $\Delta=L / K \in[0.175,0.525]$ \\
Preamble power & $\left\|\mathbf{a}_{k}\right\|_{2}^{2}=L, \forall k$ \\
Signal-to-noise ratio & SNR $=10 \mathrm{~dB}$ \\
\hline \multicolumn{2}{|c|}{ Channel } \\
\hline Channel model & Rayleigh fading \\
Noise power & $\sigma_{z}^{2}=0.1$ \\
\hline \multicolumn{2}{|c|}{ DL algorithms } \\
\hline Number of neurons (DMLP) & $N_{n}=320$ \\
Number of feature maps (CNN) & $N_{f}=40$ \\
Filter length (CNN) & $N_{w}=8$ \\
\hline \multicolumn{2}{|c|}{ LASSO [26] via CD } \\
\hline Maximum number of iterations & $T_{\mathrm{CD}}=10^{3}$ \\
Convergence criterion & $\left\|\hat{\mathbf{x}}^{t}-\hat{\mathbf{x}}^{t-1}\right\|_{2} \leq 10^{-4}$ \\
\hline \multicolumn{2}{|c|}{ AMP [27] } \\
\hline Denoising function & Complex soft thresholding [28] \\
Number of iterations & $T_{\mathrm{AmP}}=10$ \\
\hline
\end{tabular}




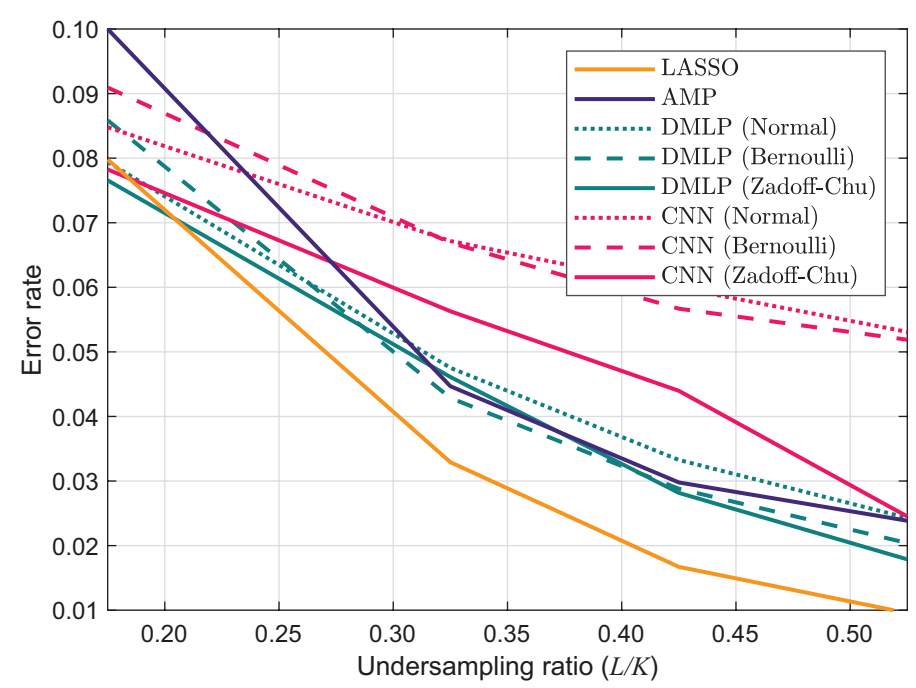

Figure 6. Detection error rate $v s$. preamble length of the DL algorithms using the three types of preamble sequences, and the baseline algorithms. $p_{a}=0.1$.

the DMLP and the CNN with ZC sequences outperform significantly the AMP algorithm. The LASSO method has the best activity detection accuracy for $\Delta>0.175$ at the expense of an extremely high computational complexity, as we demonstrate in the subsection IV-E. Moreover, the behavior of the error curves for the evaluated algorithms is similar for $p_{a}<0.1$, with decreased error rates as a consequence of the reduced number of active devices, and therefore the level of interference.

Fig. 7 depicts the ROC ${ }^{2}$ curves of the DL algorithms using the Normal, Bernoulli, and ZC sequences types for two values of undersampling ratio. For $\Delta=0.525$, the CNN using the $\mathrm{ZC}$ sequences has a huge improvement on the miss detection rate, if compared with the random sequences. Again, this is due to the correlator stage at the CNN input, the good correlation properties of the $\mathrm{ZC}$ sequences, and the $\mathrm{CNN}$ structure. Additionally, the ROC curve of the $\mathrm{CNN}$ using the $\mathrm{ZC}$ sequences is tight to the AMP algorithm. The LASSO method attains the best ROC curve among the techniques. On the other hand, for $\Delta=0.175$ the differences between the algorithms and types of sequences vanishes, as all the ROC curves of the DL algorithms are comparable to the LASSO. At the same time, the ROC of AMP degrades significantly as $\Delta$ decreases.

Fig. 8 depicts the DET $^{3}$ curves of the proposed DL algorithms and the baseline algorithms for both values of undersampling ratio. The proposed DL algorithms cover a wide range of false alarm and miss detection rates, differently from the LASSO method and the AMP algorithm. Analyzing the DET curves, we see that the algorithms achieve lower false alarm rate values than miss detection ones. Such fact is a

\footnotetext{
${ }^{2}$ The ROC curves are described by the successful detection rate, or true positive rate, $v s$. the false alarm rate, or false positive rate, allowing a comparison of the detection algorithms according to the two types of error.

${ }^{3}$ The DET curves are described by the miss detection rate $v s$. false alarm rate with the axis warped by the inverse of the Normal cumulative distribution function. The DET is more suitable than the ROC to analyze the tradeoff between the two types of error.
}

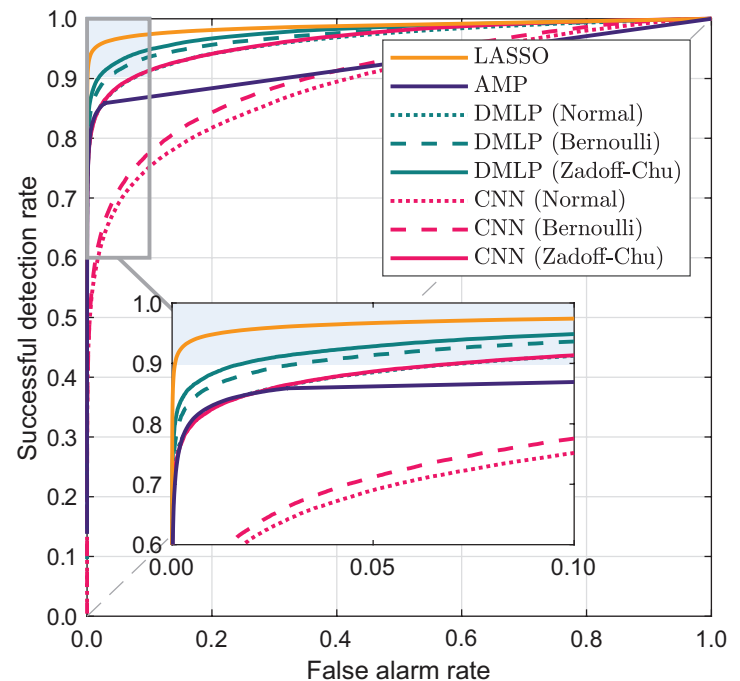

(a) $\Delta=0.525$

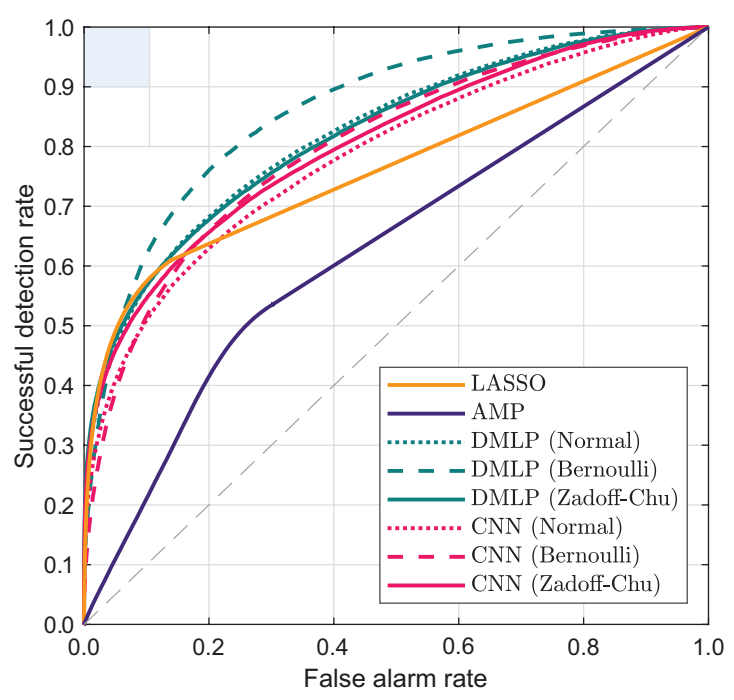

(b) $\Delta=0.175$

Figure 7. ROC curves of the DL algorithms using the three types of preamble sequences, and the baseline algorithms. $p_{a}=0.1$.

consequence of the sporadic activity of machine-type devices, which results in more inactive devices than active ones during the random access slots.

\section{Robustness of the DMLP and CNN Algorithms}

Fig. 9 depicts the error rate $v s$. the activation probability of the DL and the baseline algorithms. We evaluate each DL algorithm using the three types of sequences and two values of preamble length. Such figure of merit demonstrates the robustness of the DL activity detection algorithms against the system scenario variations, since the networks are trained for a single-fixed activation probability, $p_{a}=0.1$. As expected, the error rate increases with the activation probability, as high numbers of active devices incur in increased interference power at the received signal. Moreover, regarding the type of sequences, for $\Delta=0.525$, again the $\mathrm{ZC}$ sequences achieves the best activity detection accuracy. Both DMLP and CNN algorithms present similar results using such sequences. Next, 


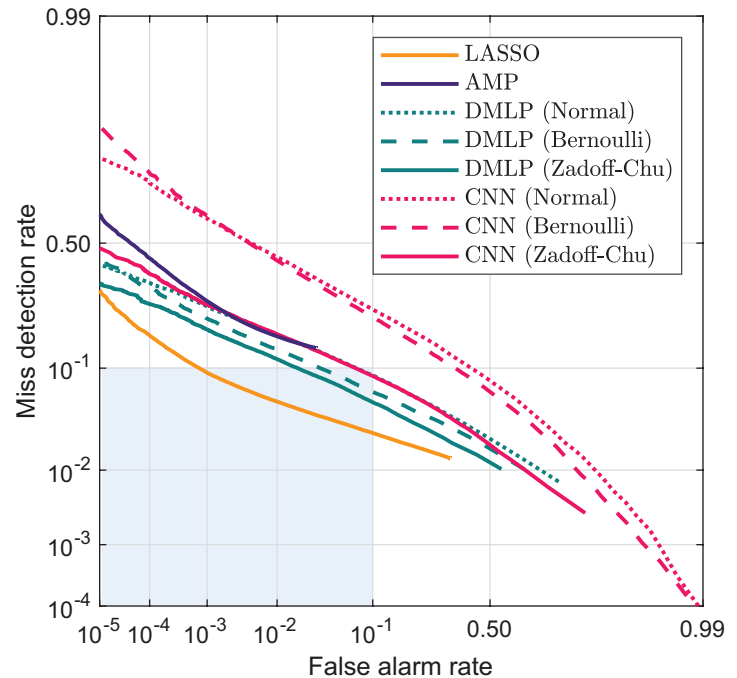

(a) $\Delta=0.525$

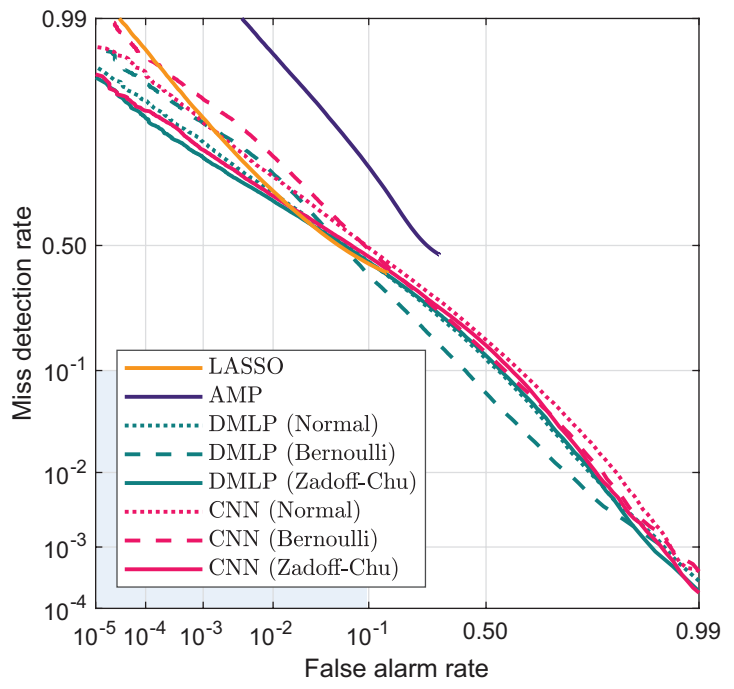

(b) $\Delta=0.175$

Figure 8. DET cuvers of the DL algorithms using the three types of preamble sequences, and the baseline algorithms. $p_{a}=0.1$.

the Bernoulli sequences outperform marginally the Normal ones. Especially, the CNN results in significant improvements using the ZC sequences, achieving detection error rates close to the DMLP. Lastly, when the undersampling ratio is reduced substantially, e.g. $\Delta=0.175$, the performance of the DMLP and the $\mathrm{CNN}$ algorithms operating under each one of the three types of sequences is tight for $p_{a} \geq 0.1$.

\section{E. Complexity of the DL and Baseline Algorithms}

Fig. 10 depicts the run time in seconds vs. the number of devices of the evaluated algorithms for two values of undersampling ratio. In this result, we set $(K, L) \in$ $\{(40,7),(40,21),(400,71),(400,211)\}$. The DL algorithms present extremely-low run time values on the order of 10 $\mu$ s to $100 \mu$ s. The DMLP algorithm has slightly shorter run time than the CNN. Compared with the baseline algorithms, the proposed algorithms result in run time values at least two

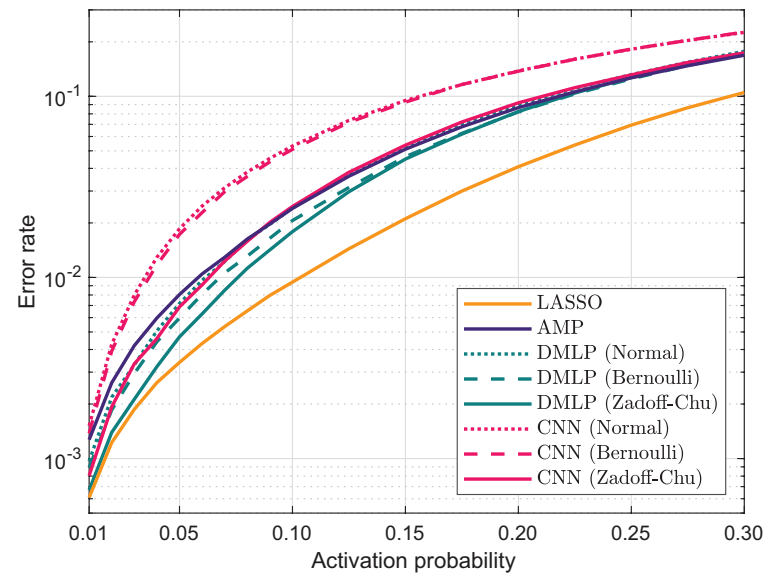

(a) $\Delta=0.525$

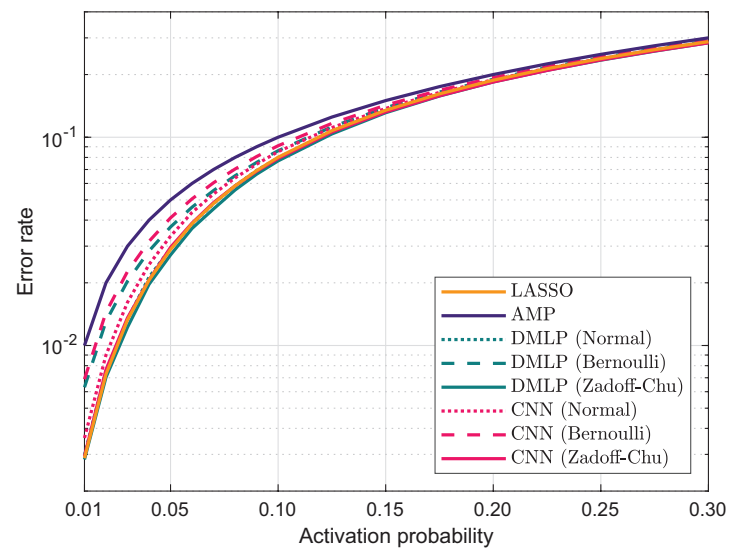

(b) $\Delta=0.175$

Figure 9. Error rate $v s$. activation probability of the DL algorithms using the three types of preamble sequences, and the baseline algorithms.

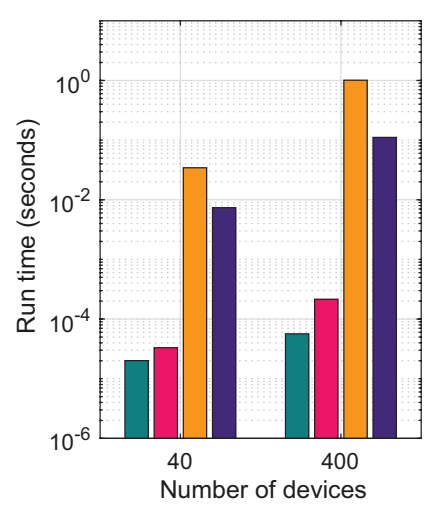

(a) $\Delta \approx 0.17$

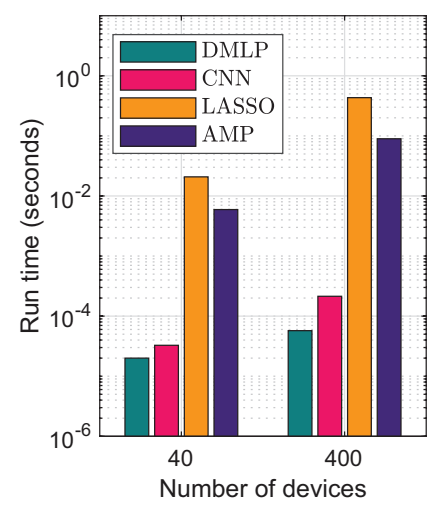

(b) $\Delta \approx 0.52$
Figure 10. Run time vs. number of devices of the DL algorithms, and the baseline algorithms. $(K, L) \in\{(40,7),(40,21),(400,71),(400,211)\}$.

orders of magnitude less. As expected, the complexity of all the algorithms increases with the number of devices, owing to the growth in the number of inputs. Despite that, the run time changes marginally with the undersampling ratio, except for the LASSO method. Such fact is due to the accelerated convergence of the $\mathrm{CD}$ algorithm caused by the amount of information provided by longer preamble sequences. 


\section{Conclusions}

In this work, we propose two DL sparse support recovery algorithms to detect active devices in GF-RA protocols. We develop a DMLP algorithm for device activity detection based on densely connected layers and a CNN algorithm built with $1 \mathrm{D}$ convolution layers. At the same time, we analyze the impact of the type of sequences used for preamble design on the activity detection accuracy. We evaluate the accuracy achieved with random preambles generated by a complex Normal and a Bernoulli distributions, as well as by deterministic $\mathrm{ZC}$ sequences. The numerical results demonstrate that the DMLP reaches the best detection error rate values among the evaluated algorithms. Despite that, the $\mathrm{CNN}$ with the ZC sequences in place of random ones achieves detection error rates comparable to the DMLP, due to the good correlation properties of these sequences and the structure of the CNN that exploits the correlation among the network inputs. On the matter of the computational complexity, both proposed DL algorithms present extremely low execution time, at the expense of the training burden to determine the suitable parameter values of the networks. Moreover, the proposed algorithms attain better performance-complexity tradeoff than state-of-art techniques, such as the LASSO method and the AMP algorithm.

\section{REFERENCES}

[1] J. Ding, D. Qu, and J. Choi, "Analysis of non-orthogonal sequences for grant-free RA with massive MIMO," IEEE Transactions on Communications, vol. 68, no. 1, pp. 150-160, Oct. 2020.

[2] X. Chen, D. W. K. Ng, W. Yu, E. G. Larsson, N. Al-Dhahir, and R. Schober, "Massive access for 5G and beyond," IEEE Journal on Selected Areas in Communications, vol. 39, no. 3, pp. 615-637, Mar. 2021.

[3] I. Goodfellow, Y. Bengio, and A. Courville, Deep Learning. Cambridge, MA, USA: MIT Press, 2016.

[4] S. Li, W. Zhang, Y. Cui, H. V. Cheng, and W. Yu, "Joint design of measurement matrix and sparse support recovery method via deep autoencoder," IEEE Signal Processing Letters, vol. 26, no. 12, pp. 17781782, Oct. 2019.

[5] W. Kim, Y. Ahn, and B. Shim, "Deep neural network-based active user detection for grant-free NOMA systems," IEEE Transactions on Communications, vol. 68, no. 4, pp. 2143-2155, Jan. 2020.

[6] N. Kim, D. Kim, B. Shim, and K. B. Lee, "Deep learning-based spreading sequence design and active user detection for massive machine-type communications," IEEE Wireless Communications Letters, vol. 10, no. 8, pp. 1618-1622, June 2021.

[7] X. Wu, S. Zhang, and J. Yan, "A CNN architecture for learning device activity from MMV," IEEE Communications Letters, vol. 25, no. 9, pp. 2933-2937, June 2021.

[8] N. Shlezinger, N. Farsad, Y. C. Eldar, and A. J. Goldsmith, "Model-based machine learning for communications," Jan. 2021, arXiv:2101.04726.

[9] M. Borgerding, P. Schniter, and S. Rangan, "AMP-inspired deep networks for sparse linear inverse problems," IEEE Transactions on Signal Processing, vol. 65, no. 16, pp. 4293-4308, May 2017.

[10] Y. Qiang, X. Shao, and X. Chen, "A model-driven deep learning algorithm for joint activity detection and channel estimation," IEEE Communications Letters, vol. 24, no. 11, pp. 2508-2512, July 2020.

[11] Y. Shi, S. Xia, Y. Zhou, and Y. Shi, "Sparse signal processing for massive device connectivity via deep learning," in 2020 IEEE International Conference on Communications Workshops (ICC Workshops), 7-11 June 2020, pp. $1-6$.

[12] W. Chen, B. Zhang, S. Jin, B. Ai, and Z. Zhong, "Solving sparse linear inverse problems in communication systems: A deep learning approach with adaptive depth," IEEE Journal on Selected Areas in Communications, vol. 39, no. 1, pp. 4-17, Jan. 2021.
[13] Y. Cui, S. Li, and W. Zhang, "Jointly sparse signal recovery and support recovery via deep learning with applications in MIMO-based grant-free random access," IEEE Journal on Selected Areas in Communications, vol. 39, no. 3, pp. 788-803, Aug. 2021.

[14] X. Shao, X. Chen, Y. Qiang, C. Zhong, and Z. Zhang, "Featureaided adaptive-tuning deep learning for massive device detection," IEEE Journal on Selected Areas in Communications, vol. 39, no. 7, pp. 18991914, May 2021.

[15] Y. Shi, H. Choi, Y. Shi, and Y. Zhou, "Algorithm unrolling for massive access via deep neural network with theoretical guarantee," IEEE Transactions on Wireless Communications, pp. 1-1, Aug. 2021.

[16] K. Gregor and Y. LeCun, "Learning fast approximations of sparse coding," in Proceedings of the 27th International Conference on International Conference on Machine Learning, 21-25 June 2010, pp. 399-406.

[17] L. Liu and W. Yu, "Massive connectivity with massive MIMO-Part I: Device activity detection and channel estimation," IEEE Transactions on Signal Processing, vol. 66, no. 11, pp. 2933-2946, Mar. 2018.

[18] K. Senel and E. G. Larsson, "Grant-free massive MTC-enabled massive MIMO: A compressive sensing approach," IEEE Transactions on Communications, vol. 66, no. 12, pp. 6164-6175, Aug. 2018.

[19] A. Fengler, S. Haghighatshoar, P. Jung, and G. Caire, "Non-bayesian activity detection, large-scale fading coefficient estimation, and unsourced random access with a massive MIMO receiver," IEEE Transactions on Information Theory, pp. 1-1, Mar. 2021.

[20] A. Mousavi and R. G. Baraniuk, "Learning to invert: Signal recovery via deep convolutional networks," in 2017 IEEE International Conference on Acoustics, Speech and Signal Processing (ICASSP), 19 June 2017, pp. 2272-2276.

[21] D. Chu, "Polyphase codes with good periodic correlation properties (corresp.)," IEEE Transactions on Information Theory, vol. 18, no. 4, pp. 531-532, July 1972.

[22] J. A. Tropp and S. J. Wright, "Computational methods for sparse solution of linear inverse problems," Proceedings of the IEEE, vol. 98, no. 6, pp. 948-958, Apr. 2010.

[23] Y. C. Eldar and G. Kutyniok, Compressed Sensing: Theory and Applications. Cambridge, Cambs., UK: Cambridge University Press, 2012.

[24] B. Popovic, "Generalized chirp-like polyphase sequences with optimum correlation properties," IEEE Transactions on Information Theory, vol. 38, no. 4, pp. 1406-1409, July 1992.

[25] K. P. Murphy, Machine Learning: A Probabilistic Perspective. Cambridge, MA, USA: The MIT Press, 2012.

[26] R. Tibshirani, "Regression shrinkage and selection via the lasso," Journal of the Royal Statistical Society: Series B (Methodological), vol. 58, no. 1, pp. 267-288, Jan. 1996.

[27] D. L. Donoho, A. Maleki, and A. Montanari, "Message-passing algorithms for compressed sensing," Proceedings of the National Academy of Sciences, vol. 106, no. 45, pp. 18 914-18 919, Sep. 2009.

[28] A. Maleki, L. Anitori, Z. Yang, and R. G. Baraniuk, "Asymptotic analysis of complex LASSO via complex approximate message passing (CAMP)," IEEE Transactions on Information Theory, vol. 59, no. 7, pp. 4290-4308, Mar. 2013.

[29] S. J. Wright, "Coordinate descent algorithms," Mathematical Programming, vol. 151, no. 1, pp. 3-34, Mar. 2015. 